\title{
Passively Mode-Locked Thulium- and Thulium/Holmium-Doped Fiber Lasers Using MXene $\mathrm{Nb}_{2} \mathrm{C}$-Coated Microfiber
}

Harith Ahmad ( $\square$ harith@um.edu.my )

University of Malaya

Rizal Ramli

University of Malaya

Nor Najwa Ismail

University of Malaya

Siti Nabila Aidit

University of Malaya

Norazriena Yusoff

University of Malaya

Muhamad Zharif Samion

University of Malaya

Research Article

Keywords: 2D, Thulium, fiber, MXene

Posted Date: February 17th, 2021

DOI: https://doi.org/10.21203/rs.3.rs-199981/v1

License: (c) (1) This work is licensed under a Creative Commons Attribution 4.0 International License.

Read Full License 


\section{Abstract}

As a result of the emergence of two-dimensional (2D) materials for various opto-electronics applications, a new class of materials named MXenes have been attracting interests due to their outstanding nonlinear properties. In this work, an MXene niobium carbide $\left(\mathrm{Nb}_{2} \mathrm{C}\right)$ was proposed and demonstrated as a saturable absorber to induce mode-locking in thulium- and thulium/holmium-doped fiber lasers. The $\mathrm{Nb}_{2} \mathrm{C}$ solution was first prepared using the liquid exfoliation technique, and then deposited onto a microfiber for integration into the laser cavity. Stable mode-locking operation was observed in both laser cavities, where the center wavelengths of the laser were recorded at $1944 \mathrm{~nm}$ for the TDFL and $1950 \mathrm{~nm}$ for the THDFL. The generated pulses in the TDFL and THDFL had repetition rates of 9.35 and $11.76 \mathrm{MHz}$ respectively, while their corresponding pulse widths were 1.67 and 1.34 ps. Both of the lasers were highly stable, having SNR values of more than $52 \mathrm{~dB}$ and showed no major fluctuations when tested for their long-term stabilities. The results demonstrate an excellent performance of the $\mathrm{Nb}_{2} \mathrm{C}$ as a saturable absorber, offering opportunities to further explore MXenes for future photonics devices.

\section{Introduction}

Optical fiber based devices are a major area of interest within the development of optical fiber amplifiers and lasers. These devices generally cover a wavelength range stretching from the S-band $(1460 \mathrm{~nm}$ to $1530 \mathrm{~nm})^{1-3}$ to L-band region (1565 nm to $\left.1625 \mathrm{~nm}\right)^{4-6}$. The advancement of optical fiber lasers has seen variety of laser output reported in a various laser configuration including pulsed ${ }^{7-12}$ and multiwavelength ${ }^{13-16}$. Tremendous achievement in the field of near-infrared (IR) fiber lasers have kept this field growing to cater the ever-growing demands from laser end-users. This include expanding the wavelength operation to $2.0 \mu \mathrm{m}$ region. Eye-safe $2.0 \mu \mathrm{m}$ laser sources are of particular interest due to its potential in medical, space, defence and industrial applications. A key aspect of $2.0 \mu \mathrm{m}$ light sources is its ability to be strongly absorbed in the human eye's vitreous part, reducing the possibility to harm the retina ${ }^{17}$. This property is much needed in free-space optical communication system ${ }^{18}$ or in LIDAR (Light Detection and Ranging) ${ }^{19-21}$. Additionally, $2.0 \mu \mathrm{m}$ laser sources play a critical role in the near-infrared (IR) gas spectroscopy and sensing owing to the efficient light absorption of atmospheric gaseous (e.g., carbon dioxide, $\mathrm{CO}_{2}$ ) at $2.0 \mu \mathrm{m}$ wavelength range ${ }^{22-24} .2 .0 \mu \mathrm{m}$ fiber laser source can be generated from thulium-doped ${ }^{25}$ and thulium/holmium-doped fiber lasers ${ }^{26}$. The thulium and thulium/holmium doped fiber, exhibit broad bandwidth covering almost $500 \mathrm{~nm}$, from $1.7 \mu \mathrm{m}$ to $2.2 \mu \mathrm{m}$. This feature allows a wide selection range of laser operations in the eye-safe spectral region, including the continuous-wave (CW) mode and the Q-switching mode. Such broad bandwidth is also known to be capable of generating femtosecond pulses in the mode-locking regime ${ }^{27-32}$.

Mode-locked fiber lasers as highly versatile light sources have attracted enormous attention due to its ability to access a wide range of scientific and industrial processes including optical communications, sensing, material processing and medical treatment ${ }^{33-38}$. So far, two main approaches utilized in the operation of mode-locked fiber lasers are based on active and passive techniques. Compared to active, 
passive technique has the intrinsic advantages of high stability and reproducibility for robust ultrashort optical pulses. Saturable absorber (SA) plays a vital role in passive mode-locking technique. Presently, SA can be classified into two broad types namely, artificial, and real. Artificial SAs such as nonlinear loop mirrors ${ }^{39-43}$ and nonlinear polarization rotation 44,45 are based on nonlinear effects with the properties of high damage threshold and low cost. These SAs provide a good platform for operation of high energy laser. However, the vulnerability of the laser system towards environmental perturbation has limit its practical applications. Real SAs, made up of materials that exhibit intensity-dependent transmission are regarded as a more effective way to generate mode-locked pulses. Specifically, two-dimensional (2D) material-based SAs have been widely employed as effective SAs due to their excellent optical properties, including wide absorption band and ultrafast recovery time. Following the successful exfoliation of graphene and its first ultrafast application, other 2D materials including topological insulators (TIs) 31,46-49, transition metal dichalcogenides (TMDs) ${ }^{50-55}$, metal chalcogenides ${ }^{56}$, antimonene ${ }^{57}$ and MXenes have been explored for their unique saturable absorption property in ultrafast laser generation.

Transition metal carbides and/or nitrides, which are widely known as MXenes, are a member of the 2D material group that possess unique properties that could be altered by simply manipulating the composition and surface termination elements ${ }^{58,59}$. In general, MXenes consist of few-atoms-thick layers of transition metal carbides, nitrides, or carbonitrides with composition of $M_{n+1} X_{n} T_{x}$, where $M$ stands for an early transition metal (such as: $\mathrm{Ti}, \mathrm{V}, \mathrm{Cr}, \mathrm{Nb}$, etc.), $\mathrm{X}$ stands for carbon and/or nitrogen, $\mathrm{n}=1,2$, or 3 , and $T_{x}$ is the surface termination groups $((-O),(-F) \text {, and }(-O H))^{60}$. Being in the family of MXene, niobium carbide $\left(\mathrm{Nb}_{2} \mathrm{C}\right)$ has received extensive research attention in the last few years due to its unique physical and chemical properties that are valuable in various applications ${ }^{61}$. Theoretically, it has been predicted that $\mathrm{Nb}_{2} \mathrm{C}$ demonstrate a great reduction of lattice thermal conductivity resulted from the abnormal electron-phonon scatterings with intensities close to that of phonon-phonon scatterings ${ }^{62}$. In a study conducted by Lin et. al. ${ }^{63}$ has revealed that the $\mathrm{Nb}_{2} \mathrm{C}$ possess strong optical response in the near infrared region as it shows high photothermal conversion efficiency that can be used in biomedicine, particularly for cancer phototherapy. In another report, Wang et. al. ${ }^{64}$ has investigated the broad-band nonlinear optical response and the ultrafast carrier dynamics of $\mathrm{Nb}_{2} \mathrm{C}$ over the wavelength ranging from visible to the near-infrared region. Their finding discloses the dependency of the nonlinear optical response of $\mathrm{Nb}_{2} \mathrm{C}$ on wavelength and excitation intensity. The unique nonlinear absorption response inversion properties of $\mathrm{Nb}_{2} \mathrm{C}$, that is the ability to shift from saturable absorption to two-photon absorption in near infrared region has facilitate its vast applications in nonlinear photonics, in particular as an optical switch ${ }^{65}$.

In this work, high-quality few layer $\mathrm{Nb}_{2} \mathrm{C}$ nanosheets were fabricated by the liquid phase exfoliation method and deposited onto a tapered fiber using a drop-casting method, forming an all-fiber SA device. Based on $\mathrm{Nb}_{2} \mathrm{C}$-coated microfiber $\mathrm{SA}$, passive mode-locking operation in TDFL and THDFL were successfully generated. Both laser systems show stable mode-locked pulses with the former system operating at $1948 \mathrm{~nm}$ and the later operating at $1952 \mathrm{~nm}$. These results suggest that the $\mathrm{Nb}_{2} \mathrm{C}$-coated microfiber could perform as a practical and a high-performance SA for an ultrafast fiber laser generation 
in the $2.0 \mu \mathrm{m}$ region. This could further promote the development of MXene-based optical devices in the photonics technology.

\section{Characterization Of Nbc Mxene}

The surface morphology of the $\mathrm{Nb}_{2} \mathrm{C} M$ Xene in the powder form was investigated by field emission scanning electron microscopy (FESEM) and the elemental composition of $\mathrm{Nb}_{2} \mathrm{C}$ MXene was determined by energy dispersive X-ray (EDX) analysis. These studies were performed using a Hitachi Model SU8220 FESEM which was equipped with EDX detector and operating at $2.0 \mathrm{kV}$. Figure 1 shows the FESEM image of $\mathrm{Nb}_{2} \mathrm{C}$ Mxene captured at the magnification of 20.0k. It can be observed that $\mathrm{Nb}_{2} \mathrm{C}$ MXene exhibits a sheet-like structure with multiple layers stacked together, whose size was about $2.0 \mu \mathrm{m}$.

Figure 2 represents the EDX elemental mapping images of $\mathrm{Nb}_{2} \mathrm{C}$ MXene where the distribution of each element can be clearly seen. The Niobium (Nb) and Carbon (C) maps verified the presence of $\mathrm{Nb}$ and $\mathrm{C}$ elements, thus confirming the successfully formation of $\mathrm{Nb}_{2} \mathrm{C}$ MXene.

An atomic force microscope (Park System NX-10 AFM) was used to measure the thickness of the $\mathrm{Nb}_{2} \mathrm{C}$ MXene. The measurement was done under non-contact mode. Initially, $10 \mu \mathrm{L}$ of $\mathrm{Nb}_{2} \mathrm{C}$ MXene solution was deposited on the Si substrate using the spin coating technique. The sample was dried at room temperature overnight before being used for thickness measurement. The AFM topography image of $\mathrm{Nb}_{2} \mathrm{C}$ MXene together with its corresponding lateral height measurement were presented in Fig. 3(a) and Fig. 3(b), respectively. The obtained result demonstrates that the $\mathrm{Nb}_{2} \mathrm{C}$ MXene exhibits few-layer structure of the $\mathrm{Nb}_{2} \mathrm{C}$ sheet with a thickness of about $28 \mathrm{~nm}$ and lateral size of approximately $1.2 \mu \mathrm{m}$.

The measurement of nonlinear optical absorption of MXene $\mathrm{Nb}_{2} \mathrm{C}$-coated microfiber $\mathrm{SA}$ was examined using the twin-detector measurement technique. A $1950 \mathrm{~nm}$ Toptica FemtoFerb femtosecond laser with a repetition rate of $30 \mathrm{MHz}$ and a pulse width of $100 \mathrm{fs}$ was employed as the seed laser. The laser source was connected to a variable attenuator and subsequently to a $3 \mathrm{~dB}$ optical coupler for beam splitting. One port of the $3 \mathrm{~dB}$ coupler was connected to the $\mathrm{Nb}_{2} \mathrm{C}$-coated microfiber $\mathrm{SA}$ and another port was connected directly to a microfiber without the SA as the reference port. Both transmitted powers were measured using optical power meter. The experimental data was recorded and fitted using the saturation model equation below:

$$
\alpha(I)=\frac{\alpha_{s}}{1+I / I_{\text {sat }}}+\alpha_{n s}
$$

where $l, l_{s a t} a_{n s}, a_{s}$ signify the laser input intensity, saturation intensity, non-saturated loss and modulation depth. From the fitting of the measured data shown in Fig. 4, the saturation intensity and modulation depth obtained were $0.4 \mathrm{MW} / \mathrm{cm} 2$ and $6.77 \%$, respectively. 


\section{Experimental Setup}

To prepare the $\mathrm{SA}$ device, the $\mathrm{Nb}_{2} \mathrm{C}$ solution was drop-casted onto a microfiber with a waist diameter and tapered length of around $8 \mu \mathrm{m}$ and $3 \mathrm{~cm}$, respectively. The insertion loss of the microfiber at $2000 \mathrm{~nm}$ was initially measured to be $6 \mathrm{~dB}$ without the $\mathrm{Nb}_{2} \mathrm{C}$, while the value increased to $7.5 \mathrm{~dB}$ after the material was deposited. The MXene-coated microfiber was incorporated into the THDFL and TDFL cavities that utilized the dual pumping mechanism as shown in Fig. 5. Both lasers had the same cavity design, except for the different active fibers being used as the amplifying medium. Two laser diodes (LDs) with center wavelengths at $1560 \mathrm{~nm}$ were used as the pump source to pump the active fiber. Each LD was then connected to a $1550 \mathrm{~nm}$ isolator to protect the LD from back-reflections. A 1550/2000 nm wavelength division multiplexer (WDM) was placed after each of the $1550 \mathrm{~nm}$ isolator to guide the $1560 \mathrm{~nm}$ pump light to the active fiber. A $2000 \mathrm{~nm}$ isolator was placed after $\mathrm{WDM}_{2}$ to allow the light to only propagate in the clockwise direction. The output of the isolator was then connected to a 90:10 coupler, where $10 \%$ of the signal was taken as the output whereas the $90 \%$ was looped back to the cavity. A polarization controller (PC) was connected to the $90 \%$ port of the coupler and was used to adjust the polarization states of the circulating light. The other end of the $\mathrm{PC}$ was connected to the $\mathrm{Nb}_{2} \mathrm{C}$-coated microfiber, then to the $2000 \mathrm{~nm}$ port of the first $\mathrm{WDM}_{1}$. Thus, completing the ring laser cavity.

The total length of the TDFL was measured around $22.1 \mathrm{~m}$, consist of $4 \mathrm{~m}$ of the TDF and $18.1 \mathrm{~m}$ single mode fiber (SMF-28), whereas the THDFL had a total cavity length of $17.4 \mathrm{~m}$, involving of $1.5 \mathrm{~m}$ of the THDF and $15.9 \mathrm{~m}$ of SMF-28. The group velocity dispersions (GVDs) of the TDF and THDF were -0.0222 $\mathrm{ps}^{2} / \mathrm{m}$ and $-0.0675 \mathrm{ps}^{2} / \mathrm{m}$, respectively. The GVD of SMF-28 was $-0.0663 \mathrm{ps}^{2} / \mathrm{m}$ at $1944 \mathrm{~nm}$ and $0.0672 \mathrm{ps}^{2} / \mathrm{m}$ at $1950 \mathrm{~nm}$. By computing the $L_{S M F-28} G V D_{S M F-28}+L_{\text {Active fiber }} G V D_{\text {Active fiber: }}$ the net cavity dispersion of $-1.28 p s^{2}$ and $-1.17 p s^{2}$ were obtained for TDFL and THDFL, respectively, indicating that the operation of both lasers were in the anomalous dispersion regime. The characteristics for the gain medium for both cavities are given in Table 1 .

Table 1

Characteristics of the active fibers used in the TDFL and THDFL cavities.

\begin{tabular}{|lll|}
\hline Cavity & $\begin{array}{l}\text { Thulium-doped Fiber } \\
\text { Laser }\end{array}$ & Thulium/Holmium-doped Fiber Laser \\
\hline Active fiber & $\begin{array}{l}\text { OFS Thulium doped fiber } \\
(\text { TmDF200) }\end{array}$ & $\begin{array}{l}\text { CorActive Thulium/Holmium doped fiber } \\
\text { (TH512) }\end{array}$ \\
\hline $\begin{array}{l}\text { Length } \\
\text { Numerical } \\
\text { aperture }\end{array}$ & 4 meter & 1.5 meter \\
\hline Absorption & 0.26 & 0.16 \\
GVD & $22 \mathrm{~dB} / \mathrm{m}^{2}$ at $1550 \mathrm{~nm}$ & $15 \mathrm{~dB} / \mathrm{m}$ at $1550 \mathrm{~nm}$ \\
\hline
\end{tabular}




\section{Results And Discussion}

\section{Mode-locked Thulium-doped fiber laser (TDFL) using $\mathrm{Nb}_{2} \mathrm{C}$.}

The performance of the $\mathrm{Nb}_{2} \mathrm{C}$ coated microfiber for the generation of mode-locking was first studied using the TDFL cavity. Mode-locked laser was initiated at pump power of $123 \mathrm{~mW}$, with a suitable adjustment of PC. Figure 6 summarizes the characteristics of mode-locked TDFL at pump power of 476 $\mathrm{mW}$. The soliton spectrum, which is a typical spectrum of mode-locked fiber laser operating in the anomalous regime, is depicted in Fig. 6(a). The spectrum was centered at $1944 \mathrm{~nm}$ with a 3-dB bandwidth of $2.8 \mathrm{~nm}$. The mode-locked pulse train is depicted in Fig. 6(b), having a repetition rate of 9.35 $\mathrm{MHz}$. The pulse train exhibits a uniform pulse intensity with a pulse interval of $107 \mathrm{~ns}$, which matched well with the cavity round-trip time. This validates the fundamental operation of the mode-locked laser. Figure 6(c) shows the autocorrelation trace fitted by a hyperbolic-secant $\left(\mathrm{Sech}^{2}\right)$ function, showing a pulse duration of $1.67 \mathrm{ps}$. With values obtained from the mode-locked, the computed time bandwidth product (TBP) is 0.320 which is marginally higher than 0.315 indicating that the pulse is slightly chirped. Figure $6(\mathrm{~d})$ shows the RF spectrum with a resolution bandwidth of $1 \mathrm{kHz}$. A distinct and sharp peak was observed at $9.35 \mathrm{MHz}$ with signal-to-noise ratio of more than $52 \mathrm{~dB}$.

\section{Mode-locked Thulium/holmium-doped fiber laser (THDFL) using $\mathrm{Nb}_{2} \mathrm{C}$.}

Subsequently, the $\mathrm{Nb}_{2} \mathrm{C}$ coated microfiber was incorporated into the THDFL. The properties of the modelocked THDFL is demonstrated in Fig. 7 at pump power of $397 \mathrm{~mW}$. Figure 7(a) illustrates the modelocked THDFL spectrum, having a center wavelength at $1950.8 \mathrm{~nm}$ with a full-width at half maximum (FWHM) of $3 \mathrm{~nm}$. Similar to the output spectrum of the TDFL, Kelly sidebands were also observed in the optical spectrum. This was the result of the gain fibers exhibiting a relatively large anomalous dispersion in the $2.0 \mu \mathrm{m}$ region, causing the mode-locking operation of the $2.0 \mu \mathrm{m}$ fiber lasers often lying in the conventional soliton regime. Figure 7(b) shows the mode-locked pulse train having a pulse interval of 85 $\mathrm{ns}$, with a corresponding repetition rate of $11.76 \mathrm{MHz}$. This tallies well with the fundamental frequency estimated from the total cavity length. The pulse profile obtained from the autocorrelation measurement was fitted with a sech ${ }^{2}$ profile, as illustrated in Fig. 7(c). The pulse width verified with fitted function is around 1.34 ps. The TBP was estimated to be 0.317 , which was only slightly larger than the TBP limit of 0.315. This indicates that the generated pulses were close to their transform-limited operation. The RF spectrum was also measured and shown in Fig. 7(d). A distinct peak was observed at the fundamental frequency of $11.76 \mathrm{MHz}$ with an SNR of more than $64 \mathrm{~dB}$. Additionally, the RF spectrum on a wider range of $400 \mathrm{MHz}$ shown in the inset of Fig. 7(d) was seen to be free from any spectral modulation, which eliminates possible Q-switching instabilities.

Figure 8 shows the variation of average output power against pump power for both mode-locked TDFL and THDFL cavities. Low mode-locking threshold of $123 \mathrm{~mW}$ was observed in the TDFL. At maximum pump power of $476 \mathrm{~mW}$, the maximum output power for the mode-locked TDFL was recorded to be around $1.1 \mathrm{~mW}$. The slope efficiency of TDFL is $0.3 \%$. The corresponding peak power was calculated 
around $70 \mathrm{~W}$. Meanwhile, the mode-locked THDFL had a maximum output power of around $4.6 \mathrm{~mW}$ at maximum pump power of $397 \mathrm{~mW}$ with corresponding pulse peak power of $291 \mathrm{~W}$. As compared to TDFL, higher slope efficiency of $1.7 \%$ was obtained in THDFL. It should be noted that a harmonic modelocking operation was obtained at pump power greater than $397 \mathrm{~mW}$, nevertheless it was not reported here as the interest of this work is only fundamental mode-locking operation. Both output power could be linearly fitted with minimal error, suggesting the capability of the lasers to generate a higher output power if a higher pump power were available.

The $\mathrm{Nb}_{2} \mathrm{C}$-coated microfiber $\mathrm{SA}$ also exhibited an excellent stability against degradation, maintaining stable mode-locking operation when tested for its long-term stability. This can be seen from Fig. 9, where the mode-locked spectrum of TDFL and THDFL was recorded at every 10 minutes for 1 -hour. Throughout the stability measurement, the center wavelengths of the lasers were maintained at $1944 \mathrm{~nm}$ and 1950.8 $\mathrm{nm}$ for the TDFL and the THDFL, respectively. Additionally, no significant power fluctuation was observed during their operations. To further verify the generation of mode-locked pulses in both laser cavities were induced by $\mathrm{Nb}_{2} \mathrm{C}$-coated microfiber $\mathrm{SA}$, the $\mathrm{SA}$ device was removed from the laser cavities. At any pump power level, only continuous-wave (CW) laser operation was observed in both TDFL and THDFL. This proves that the mode-locking operation was induced by the saturable absorption effect from the $\mathrm{Nb}_{2} \mathrm{C}$ completely.

\section{Methods}

\section{Preparation of $\mathrm{Nb}_{2} \mathrm{C}$ MXene.}

Initially, $50 \mathrm{mg}$ of $\mathrm{Nb}_{2} \mathrm{C}$ powder (2D Semiconductors, USA) was added in a glass vial containing $10 \mathrm{~mL}$ of isopropyl alcohol (IPA, Sigma Aldrich). The mixture was put under the sonication process using a Hielscher UP200Ht probe type sonicator to obtain a homogenous solution. The sonicator was operated with 5 seconds on pulse, 5 seconds off pulse and $40 \%$ power for 4 hours. Next, the obtained black colour suspension was centrifuged for 10 minutes at $4000 \mathrm{rpm}$ to separate the undissolved powder. The supernatant was collected for further use and has been labelled as $\mathrm{Nb}_{2} \mathrm{C}$ Mxene solution.

\section{Laser characterisation.}

To analyse the output mode-locked pulses, a $2 \mu \mathrm{m}$ Newport 818-BB-51F photodetector was used together with a Yogokawa DLM2054 oscilloscope and a Rohde \& Schwarz FPC1000 radio-frequency spectrum analyser (RFSA). The spectrum of the mode-locked laser was recorded using a Yokogawa AQ6375 optical spectrum analyser (OSA) at the highest resolution of $0.05 \mathrm{~nm}$. The pulse width measurement was measured by an A.P.E Pulsecheck 150 autocorrelator while the output power was measured using a Thorlabs S148C optical power meter (OPM).

\section{Conclusion}


An MXene niobium carbide $\left(\mathrm{Nb}_{2} \mathrm{C}\right)$ was successfully proven as a saturable absorber to produce stable

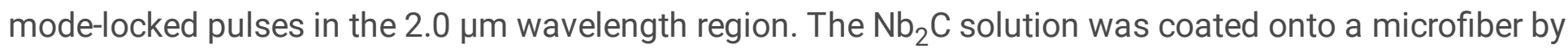
the drop-cast method to allow easy integration into thulium- and thulium/holmium-doped fiber laser cavities. The TDFL had a center wavelength at $1944 \mathrm{~nm}$, while the center wavelength of the THDFL was recorded at a slightly longer wavelength at $1950 \mathrm{~nm}$. The generated pulses in the TDFL and THDFL had repetition rates of 9.35 and $11.76 \mathrm{MHz}$, respectively, while their corresponding pulse widths were 1.67 and $1.34 \mathrm{ps}$. The maximum peak power generated was $70 \mathrm{~W}$ for the TDFL and as high as $291 \mathrm{~W}$ for the THDFL. The lasers were highly stable when tested for their long-term stabilities, where no major fluctuation in the center wavelength as well as the peak optical power was observed. The stability was further proven with the SNR values of both lasers recorded to be more than $52 \mathrm{~dB}$. The results show the use of the $\mathrm{Nb}_{2} \mathrm{C}$ as a promising mode-locker, offering opportunities to further explore the use of MXenes for future photonics devices.

\section{Declarations}

\section{Acknowledgment}

This work is funded by the University of Malaya under the grants RK021-2019, TOP100PRC and RU0022020 and the Ministry of Higher Education, Malaysia under the grant HiCoE Phase II.

\section{Author information}

\section{Affiliations}

Photonics Research Centre, University of Malaya, 50603 Kuala Lumpur, Malaysia.

Harith Ahmad, Rizal Ramli, Nor Najwa Ismail, Siti Nabila Aidit, Norazriena Yusoff and Muhamad Zharif Samion.

Physics Department, Faculty of Science, University of Malaya, 50603 Kuala Lumpur, Malaysia.

Harith Ahmad

\section{Author contributions statements}

Harith Ahmad proposed the study and designed the experiment. Rizal Ramli performed the TDFL experiment. Nor Najwa Ismail conducted the THDFL experiment. Siti Nabila Aidit contributed to writing of the manuscript. Norazriena Yusoff prepared the $\mathrm{Nb}_{2} \mathrm{C}$ MXene, performed the material characterization and contributed to writing of the manuscript. Muhamad Zharif Samion contributed to data analysis.

\section{Competing interests}

The authors declare no competing financial interests. 
Correspondence and requests for materials should be addressed to H.A.

\section{References}

1. Harun, S. W., Saat, N. K. \& Ahmad, H. An efficient S-band erbium-doped fiber amplifier using doublepass configuration. IEICE Electron. Express 2, 182-185 (2005).

2. Rosolem, J. B. et al. All silica S-band double-pass erbium-doped fiber amplifier. IEEE Photonics Technol. Lett. 17, 1399-1401 (2005).

3. Tanabe, S. \& Tamaoka, T. Gain characteristics of Tm-doped fluoride fiber amplifier in S-band by dualwavelength pumping. J. Non. Cryst. Solids 326, 283-286 (2003).

4. Harun, S. W., Low, S. K., Poopalan, P. \& Ahmad, H. Gain clamping in L-band erbium-doped fiber amplifier using a fiber Bragg grating. IEEE photonics Technol. Lett. 14, 293-295 (2002).

5. Yang, J., Meng, X. \& Liu, C. Accurately control and flatten gain spectrum of L-band erbium doped fiber amplifier based on suitable gain-clamping. Opt. Laser Technol. 78, 74-78 (2016).

6. Ahmad, H., Shahi, S. \& Harun, S. W. Bismuth-based erbium-doped fiber as a gain medium for L-band amplification and Brillouin fiber laser. Laser Phys. 20, 716-719 (2010).

7. Xu, N., Ming, N., Han, X., Man, B. \& Zhang, H. Large-energy passively Q-switched Er-doped fiber laser based on $\mathrm{CVD}-\mathrm{Bi}_{2} \mathrm{Se}_{3}$ as saturable absorber. Opt. Mater. Express 9, 373-383 (2019).

8. Liu, J. et al. Switchable dual-wavelength Q-switched fiber laser using multilayer black phosphorus as a saturable absorber. Photonics Res. 6, 198-203 (2018).

9. Liu, L. et al. Passively Q-switched pulses generation from Erbium-doped fiber laser based on microfiber coated PbS quantum dots. Opt. Fiber Technol. 46, 162-166 (2018).

10. Luo, X. et al. All-Fiber Mode-Locked Laser Based on Mamyshev Mechanism With High-Energy Pulse Generation at $1550 \mathrm{~nm}$. J. Light. Technol. 38, 1468-1473 (2020).

11. Elahi, P., Kalaycıoğlu, H., Li, H., Akçaalan, Ö. \& Ilday, F. Ö. 175 fs-long pulses from a high-power singlemode Er-doped fiber laser at 1550 nm. Opt. Commun. 403, 381-384 (2017).

12. Li, D. et al. Wavelength and pulse duration tunable ultrafast fiber laser mode-locked with carbon nanotubes. Sci. Rep. 8, 1-8 (2018).

13. Parvizi, R., Arof, H., Ali, N. M., Ahmad, H. \& Harun, S. W. $0.16 \mathrm{~nm}$ spaced multi-wavelength Brillouin fiber laser in a figure-of-eight configuration. Opt. Laser Technol. 43, 866-869 (2011).

14. Ahmad, H., Zulkifli, M. Z., Latif, A. A. \& Harun, S. W. Tunable dual wavelength fiber laser incorporating AWG and optical channel selector by controlling the cavity loss. Opt. Commun. 282, 4771-4775 (2009).

15. Zhou, Y., Lou, S., Tang, Z., Zhao, T. \& Zhang, W. Tunable and switchable C-band and L-band multiwavelength erbium-doped fiber laser employing a large-core fiber filter. Opt. Laser Technol. 111, 262270 (2019). 
16. He, W., Zhu, L., Meng, F., Song, Y. \& Dong, M. Selectable and stable C-band multi-wavelength ring cavity erbium-doped fiber laser employing Sagnac loop and tunable filter. Optik (Stuttg). 176, 528534 (2019).

17. Scholle, K., Lamrini, S., Koopmann, P. \& Fuhrberg, P. 2 um laser sources and their possible applications. in Frontiers in guided wave optics and optoelectronics (IntechOpen, 2010).

18. Wilkins, G. D. Eye-safe free-space laser communications. in Proceedings of the IEEE National Aerospace and Electronics Conference NAECON 1996 vol. 2 710-715 (1996).

19. Singh, U. N., Yu, J., Petros, M., Refaat, T. \& Reithmaier, K. Development of a pulsed 2-micron integrated path differential absorption lidar for $\mathrm{CO}_{2}$ measurement. in Lidar Remote Sensing for Environmental Monitoring XIV vol. 8872887209 (International Society for Optics and Photonics, 2013).

20. Singh, U. N. et al. Airborne 2-micron double-pulsed integrated path differential absorption lidar for column $\mathrm{CO}_{2}$ measurement. in Lidar Technologies, Techniques, and Measurements for Atmospheric Remote Sensing X vol. 9246924602 (International Society for Optics and Photonics, 2014).

21. Mizutani, K. et al. Conductive-cooled 2-micron laser development for wind and $\mathrm{CO}_{2}$ measurements. in Lidar Remote Sensing for Environmental Monitoring XIII vol. 8526852603 (International Society for Optics and Photonics, 2012).

22. Stepanov, E. V, Kotelnikov, S. N., Stavtsev, A. Y. \& Kasoev, S. G. The best absorption lines for the detection of carbon monoxide at 2.35 micron with tunable diode lasers. in Journal of Physics: Conference Series vol. 156012053 (IOP Publishing, 2020).

23. McAleavey, F. J. et al. Narrow linewidth, tunable $\mathrm{Tm}^{3+}$-doped fluoride fiber laser for optical-based hydrocarbon gas sensing. IEEE J. Sel. Top. quantum Electron. 3, 1103-1111 (1997).

24. Ghosh, A., Roy, A. S., Chowdhury, S. Das, Sen, R. \& Pal, A. All-fiber tunable ring laser source near $2 \mu \mathrm{m}$ designed for $\mathrm{CO}_{2}$ sensing. Sensors Actuators B Chem. 235, 547-553 (2016).

25. Li, Z. et al. Thulium-doped fiber amplifier for optical communications at $2 \mu \mathrm{m}$. Opt. Express 21, 9289-9297 (2013).

26. Honzatko, P., Baravets, Y., Kasik, I. \& Podrazky, O. Wideband thulium-holmium-doped fiber source with combined forward and backward amplified spontaneous emission at 1600-2300 nm spectral band. Opt. Lett. 39, 3650-3653 (2014).

27. Wang, J. et al. 152 fs nanotube-mode-locked thulium-doped all-fiber laser. Sci. Rep. 6, 1-7 (2016).

28. Sun, B. et al. 1867-2010 nm tunable femtosecond thulium-doped all-fiber laser. Opt. Express 25, 8997-9002 (2017).

29. Meng, Y., Li, Y., Xu, Y. \& Wang, F. Carbon nanotube mode-locked thulium fiber laser with $200 \mathrm{~nm}$ tuning range. Sci. Rep. 7, 1-7 (2017).

30. Hoogland, H., Hänsel, W. \& Holzwarth, R. Novel Robust 2- $\mu$ m All-PM Thulium/Holmium Based Femtosecond Fiber Laser Oscillator. in CLEO: Science and Innovations SM3L-4 (Optical Society of America, 2017). 
31. Lee, J. \& Lee, J. H. Femtosecond Tm-Ho co-doped fiber laser using a bulk-structured $\mathrm{Bi}_{2} \mathrm{Se}_{3}$ topological insulator. Chinese Phys. B 27, 94219 (2018).

32. Jung, M. et al. A femtosecond thulium holmium co-doped fiber laser based on graphene oxide evanescent filed interaction. in 2014 Conference on Lasers and Electro-Optics (CLEO)-Laser Science to Photonic Applications 1-2 (IEEE, 2014).

33. Whitenett, G., Stewart, G., Yu, H. \& Culshaw, B. Investigation of a tuneable mode-locked fiber laser for application to multipoint gas spectroscopy. J. Light. Technol. 22, 813 (2004).

34. Mingareev, I. et al. Welding of polymers using a $2 \mu \mathrm{m}$ thulium fiber laser. Opt. Laser Technol. 44, 2095-2099 (2012).

35. Fried, N. M. \& Murray, K. E. High-power thulium fiber laser ablation of urinary tissues at $1.94 \mu \mathrm{m} . \mathrm{J}$. Endourol. 19, 25-31 (2005).

36. Marcinkevičius, A. et al. Femtosecond laser-assisted three-dimensional microfabrication in silica. Opt. Lett. 26, 277-279 (2001).

37. Kondo, Y. et al. Fabrication of long-period fiber gratings by focused irradiation of infrared femtosecond laser pulses. Opt. Lett. 24, 646-648 (1999).

38. Chou, S. Y., Keimel, C. \& Gu, J. Ultrafast and direct imprint of nanostructures in silicon. Nature 417, 835-837 (2002).

39. Runge, A. F. J., Aguergaray, C., Provo, R., Erkintalo, M. \& Broderick, N. G. R. All-normal dispersion fiber lasers mode-locked with a nonlinear amplifying loop mirror. Opt. Fiber Technol. 20, 657-665 (2014).

40. Kim, D., Kwon, D., Lee, B. \& Kim, J. Polarization-maintaining nonlinear-amplifying-loop-mirror modelocked fiber laser based on a 3×3 coupler. Opt. Lett. 44, 1068-1071 (2019).

41. Szczepanek, J., Kardaś, T. M., Michalska, M., Radzewicz, C. \& Stepanenko, Y. Simple all-PM-fiber laser mode-locked with a nonlinear loop mirror. Opt. Lett. 40, 3500-3503 (2015).

42. Ou, S., Liu, G., Qiu, D., Guo, L. \& Zhang, Q. All-polarization-maintaining, all-normal-dispersion modelocked fiber laser with spectral filtering in a nonlinear optical loop mirror. Opt. Express 28, 2367023678 (2020).

43. Chernysheva, M. A., Krylov, A. A., Kryukov, P. G. \& Dianov, E. M. Nonlinear Amplifying Loop-MirrorBased Mode-Locked Thulium-Doped Fiber Laser. IEEE Photonics Technol. Lett. 24, 1254-1256 (2012).

44. Gao, C., Wang, Z., Luo, H. \& Zhan, L. High energy all-fiber Tm-doped femtosecond soliton laser modelocked by nonlinear polarization rotation. J. Light. Technol. 35, 2988-2993 (2017).

45. Szczepanek, J., Kardaś, T. M., Radzewicz, C. \& Stepanenko, Y. Ultrafast laser mode-locked using nonlinear polarization evolution in polarization maintaining fibers. Opt. Lett. 42, 575-578 (2017).

46. Yin, K. et al. Soliton mode-locked fiber laser based on topological insulator $\mathrm{Bi}_{2} \mathrm{Te}_{3}$ nanosheets at 2 um. Photonics Res. 3, 72-76 (2015).

47. Sotor, J., Sobon, G., Macherzynski, W. \& Abramski, K. M. Harmonically mode-locked Er-doped fiber laser based on $\mathrm{S} \mathrm{Sb}_{2} \mathrm{Te}_{3}$ topological insulator saturable absorber. Laser Phys. Lett. 11, 55102 (2014). 
48. Sotor, J., Sobon, G. \& Abramski, K. M. Sub-130 fs mode-locked Er-doped fiber laser based on topological insulator. Opt. Express 22, 13244-13249 (2014).

49. Liu, W. et al. 70-fs mode-locked erbium-doped fiber laser with topological insulator. Sci. Rep. 6, 19997 (2016).

50. Li, L., Pang, L., Zhao, Q., Liu, W. \& Su, Y. VSe 2 nanosheets for ultrafast fiber lasers. J. Mater. Chem. C 8, 1104-1109 (2020).

51. Cheng, P. K. et al. Ultrafast Yb-Doped Fiber Laser Using Few Layers of PdS 2 Saturable Absorber. Nanomaterials 10, 2441 (2020).

52. Liu, W., Liu, M., Liu, X., Lei, M. \& Wei, Z. SnSSe as a saturable absorber for an ultrafast laser with superior stability. Opt. Lett. 45, 419-422 (2020).

53. Lee, J., Koo, J., Lee, J., Jhon, Y. M. \& Lee, J. H. All-fiberized, femtosecond laser at 1912 nm using a bulk-like $\mathrm{MoSe}_{2}$ saturable absorber. Opt. Mater. Express 7, 2968-2979 (2017).

54. Liu, W. et al. Tungsten disulfide saturable absorbers for 67 fs mode-locked erbium-doped fiber lasers. Opt. Express 25, 2950-2959 (2017).

55. Liu, W. et al. Tungsten diselenide for mode-locked erbium-doped fiber lasers with short pulse duration. Nanotechnology 29, 174002 (2018).

56. Feng, J. et al. 2D ductile transition metal chalcogenides (TMCs): novel high-performance $\mathrm{Ag}_{2} \mathrm{~S}$ nanosheets for ultrafast photonics. Adv. Opt. Mater. 8, 1901762 (2020).

57. Song, Y. et al. Few-layer antimonene decorated microfiber: ultra-short pulse generation and all-optical thresholding with enhanced long term stability. 2D Mater. 4, 45010 (2017).

58. Ahmad, H. et al. Tunable passively Q-switched erbium-doped fiber laser based on $\mathrm{Ti}_{3} \mathrm{C}_{2} \mathrm{~T}_{\mathrm{x}} \mathrm{MXene}$ as saturable absorber. Opt. Fiber Technol. 58, 102287 (2020).

59. Hart, J. L. et al. Control of MXenes' electronic properties through termination and intercalation. Nat. Commun. 10, 1-10 (2019).

60. Gogotsi, Y. \& Anasori, B. The rise of MXenes. ACS Nano 13, 8491-8494 (2019).

61. Gao, L. et al. Applications of Few-Layer $\mathrm{Nb}_{2} \mathrm{C}$ MXene: Narrow-Band Photodetectors and Femtosecond Mode-Locked Fiber Lasers. ACS Nano 15, 954-965 (2021)

62. Huang, Y., Zhou, J., Wang, G. \& Sun, Z. Abnormally Strong Electron-Phonon Scattering Induced Unprecedented Reduction in Lattice Thermal Conductivity of Two-Dimensional Nb ${ }_{2}$ C. J. Am. Chem. Soc. 141, 8503-8508 (2019).

63. Lin, H., Gao, S., Dai, C., Chen, Y. \& Shi, J. A two-dimensional biodegradable niobium carbide (MXene) for photothermal tumor eradication in NIR-I and NIR-II biowindows. J. Am. Chem. Soc. 139, 1623516247 (2017).

64. Wang, Y. et al. Niobium carbide MXenes with broad-band nonlinear optical response and ultrafast carrier dynamics. ACS Nano 14, 10492-10502 (2020). 
65. Gao, L. et al. Ultrafast Relaxation Dynamics and Nonlinear Response of Few-Layer Niobium Carbide MXene. Small Methods 4, 2000250 (2020).

\section{Figures}

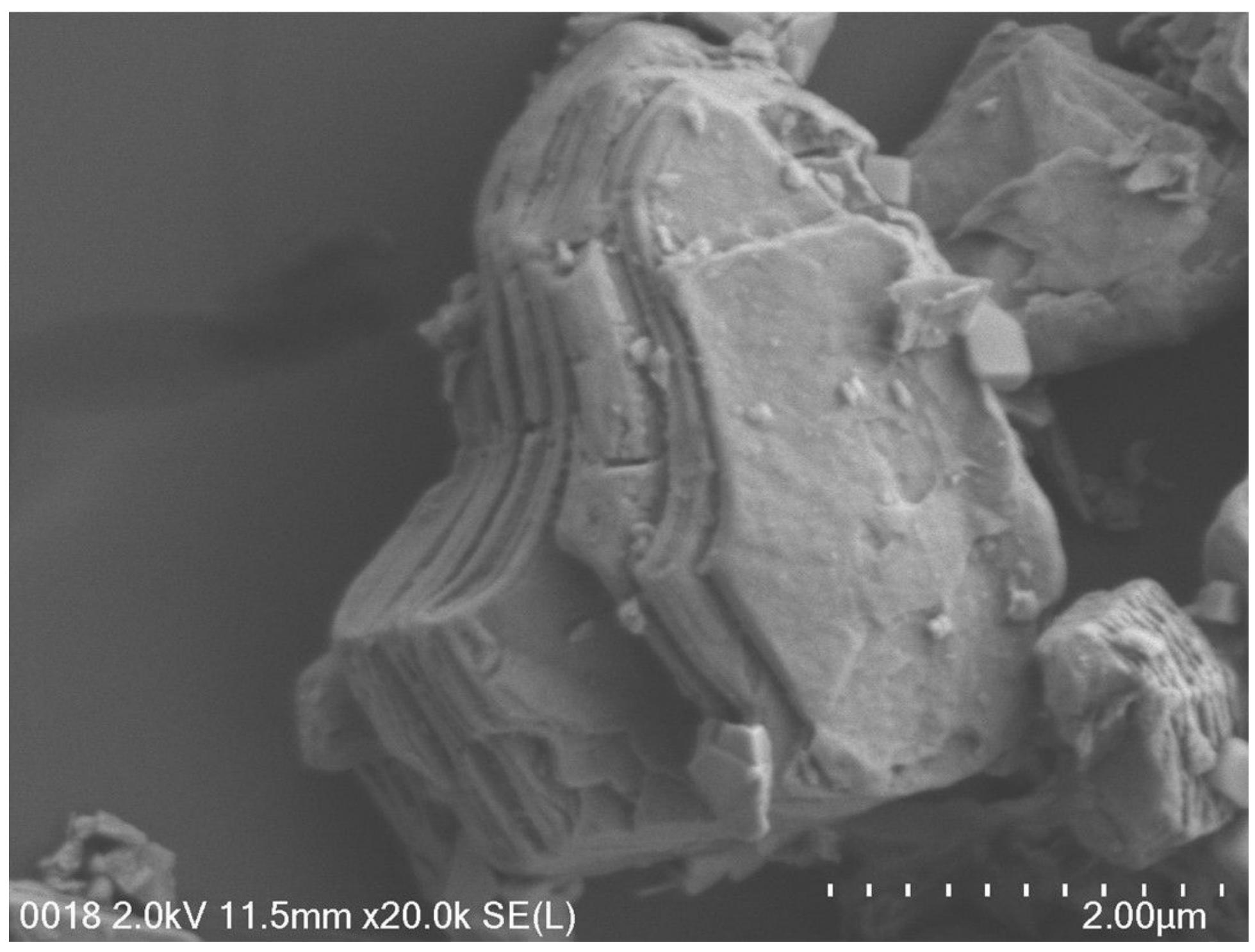

\section{Figure 1}

FESEM image of Nb2C MXene in the powder form. 


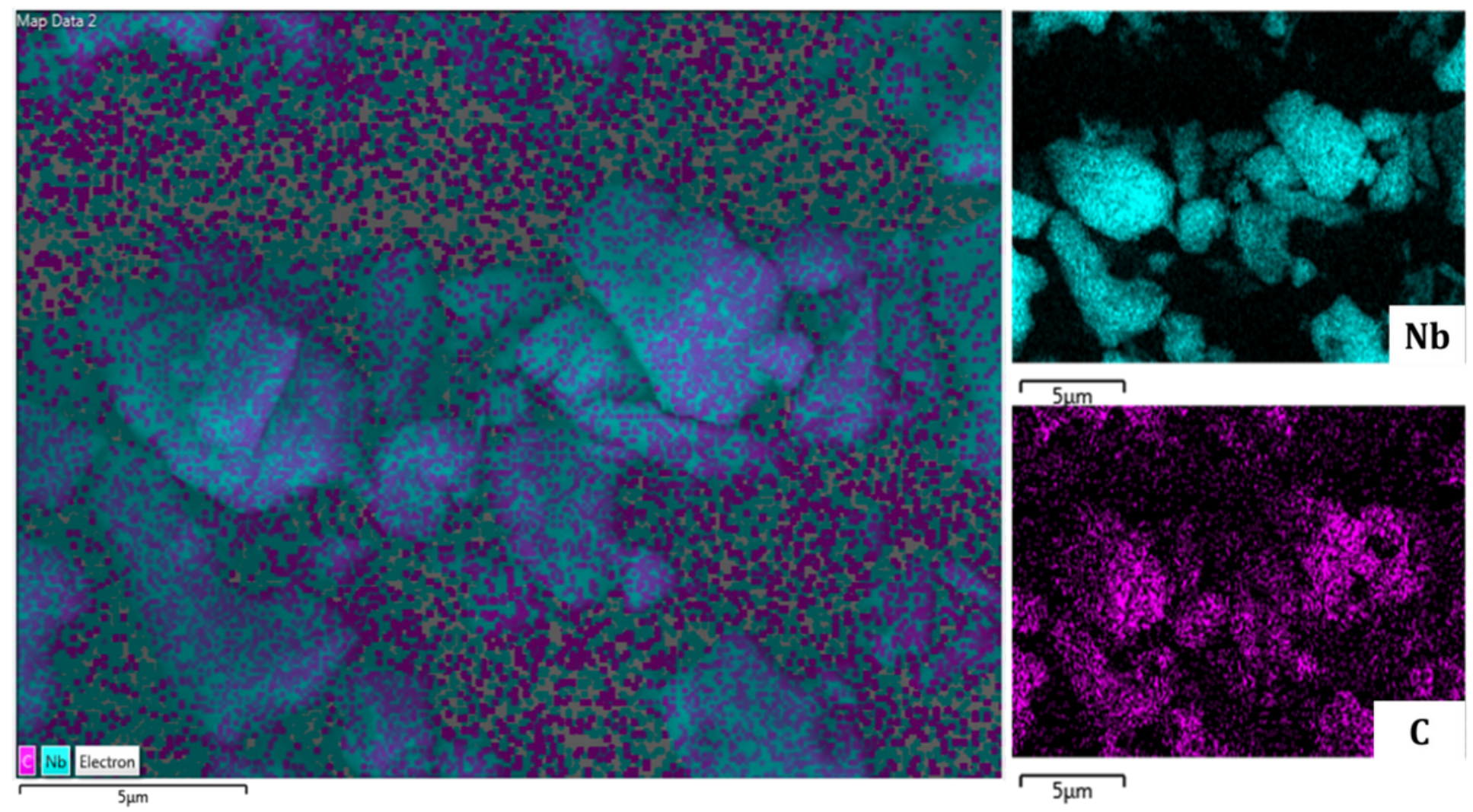

Figure 2

EDX elemental mapping of $\mathrm{Nb} 2 \mathrm{C}$ MXene. 
$\mathrm{nm}$

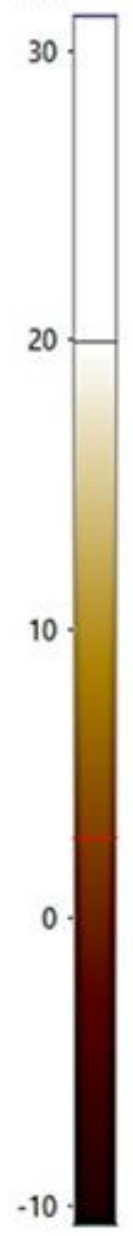

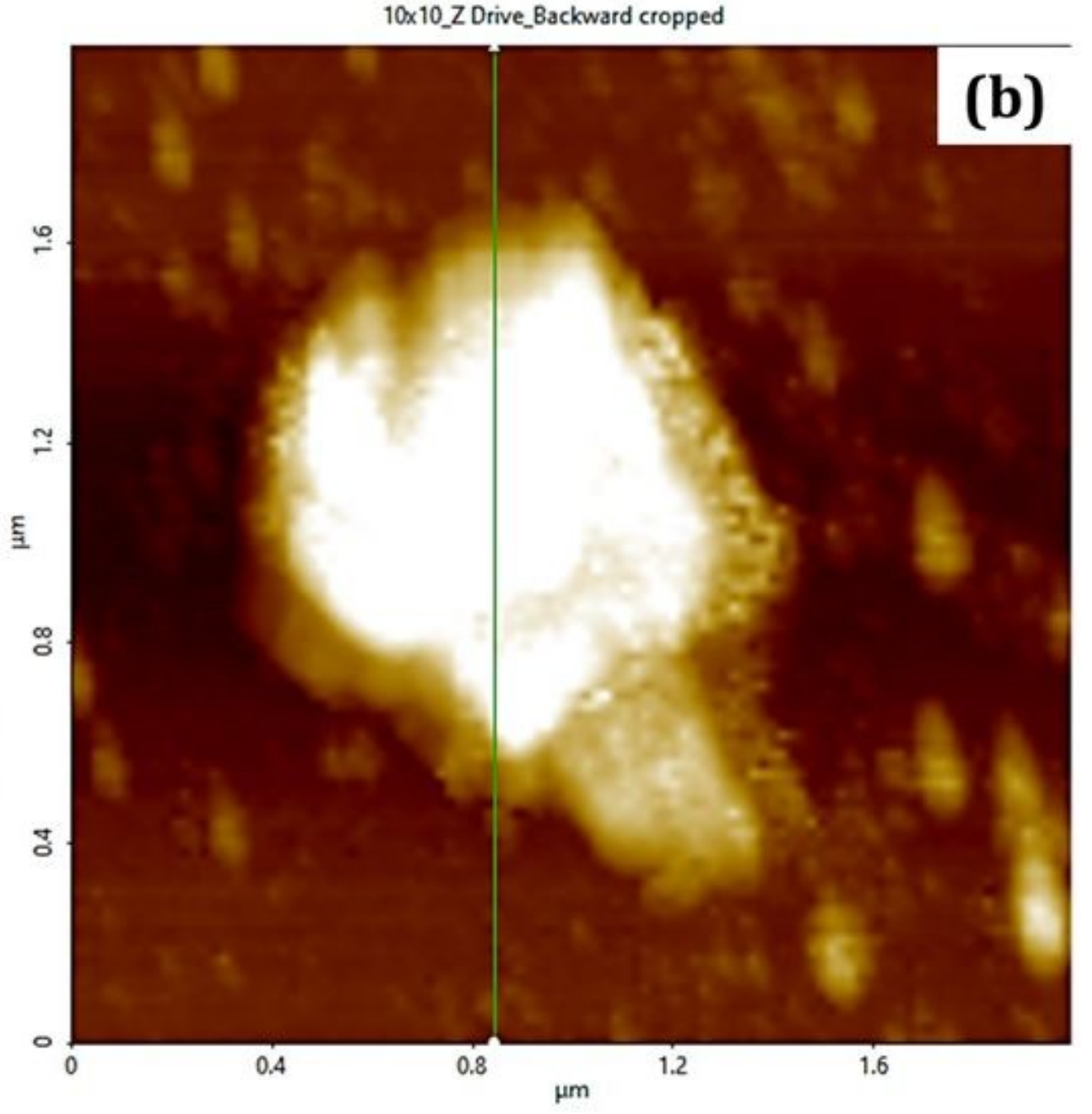

Line Profile Green - 44

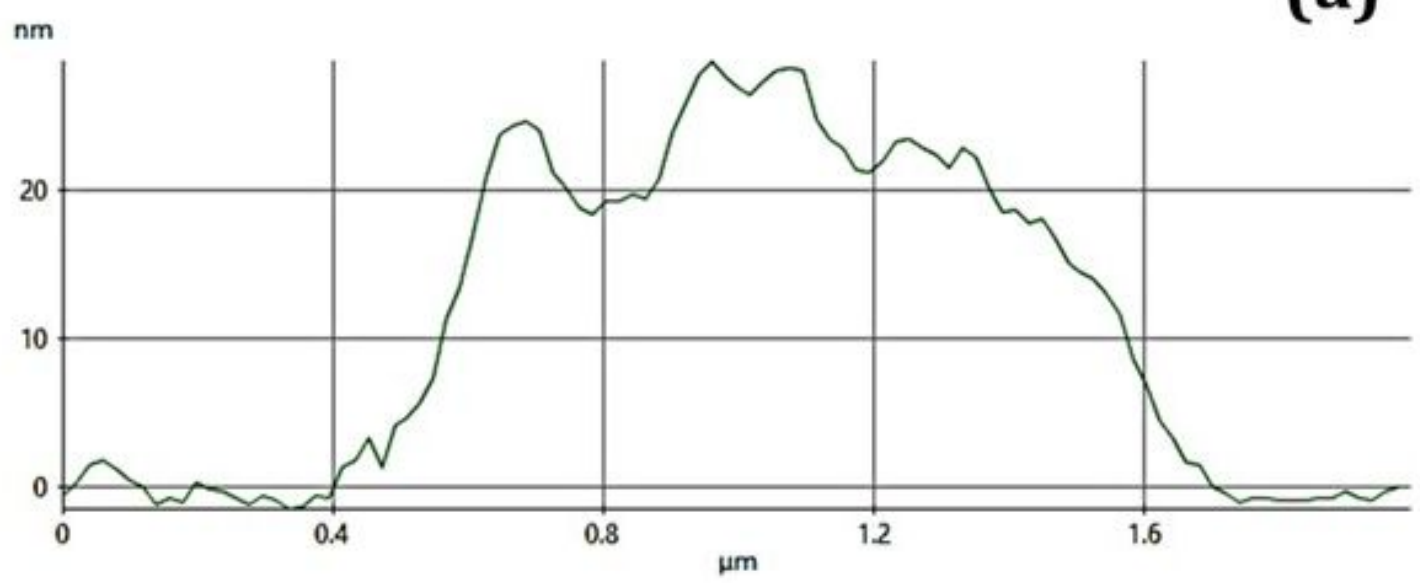

Figure 3

(a) AFM topography image of $\mathrm{Nb2C}$ MXene and (b) the corresponding lateral height measurement. 


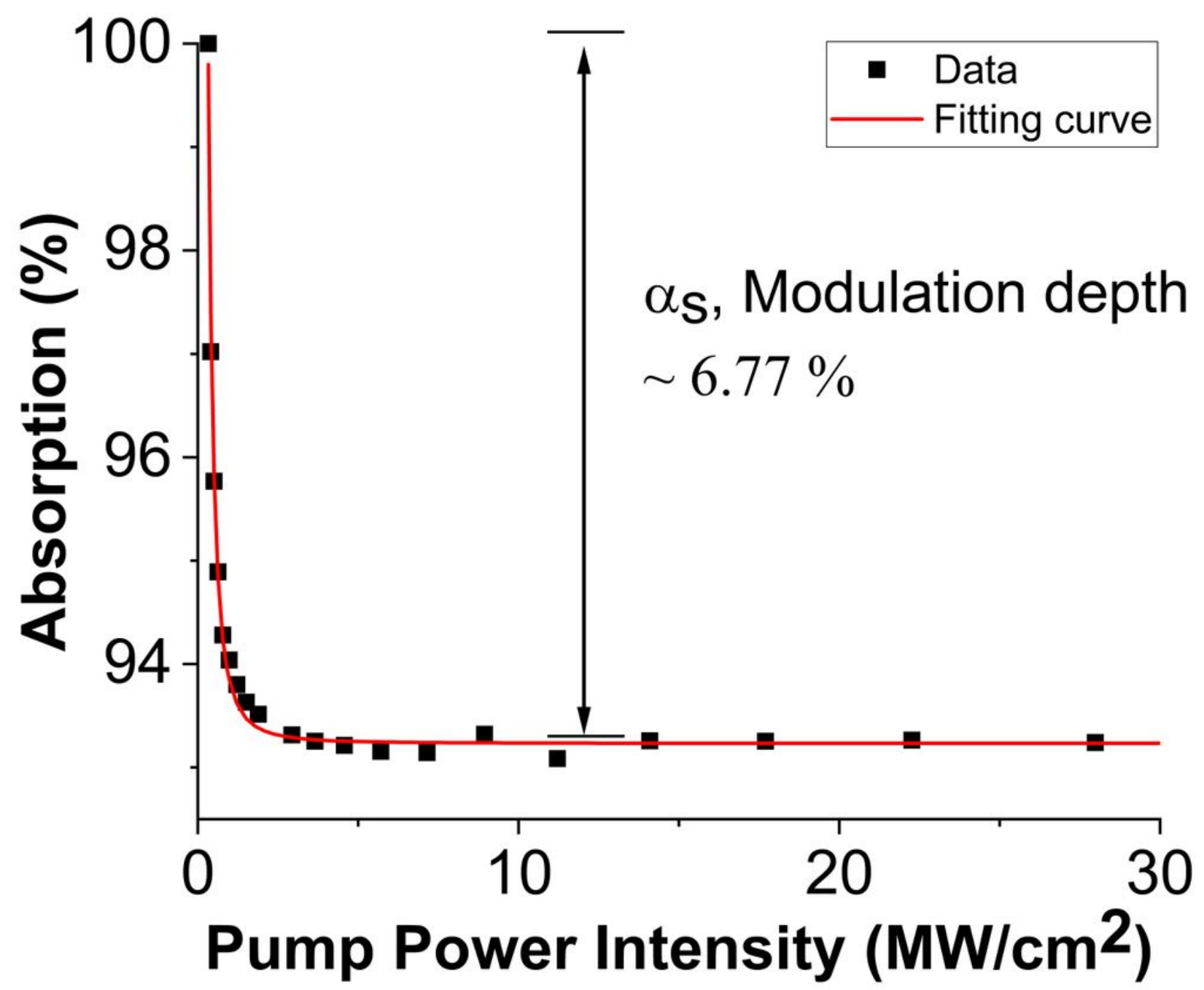

Figure 4

Nonlinear absorption curve of MXene Nb2C-coated microfiber SA. 


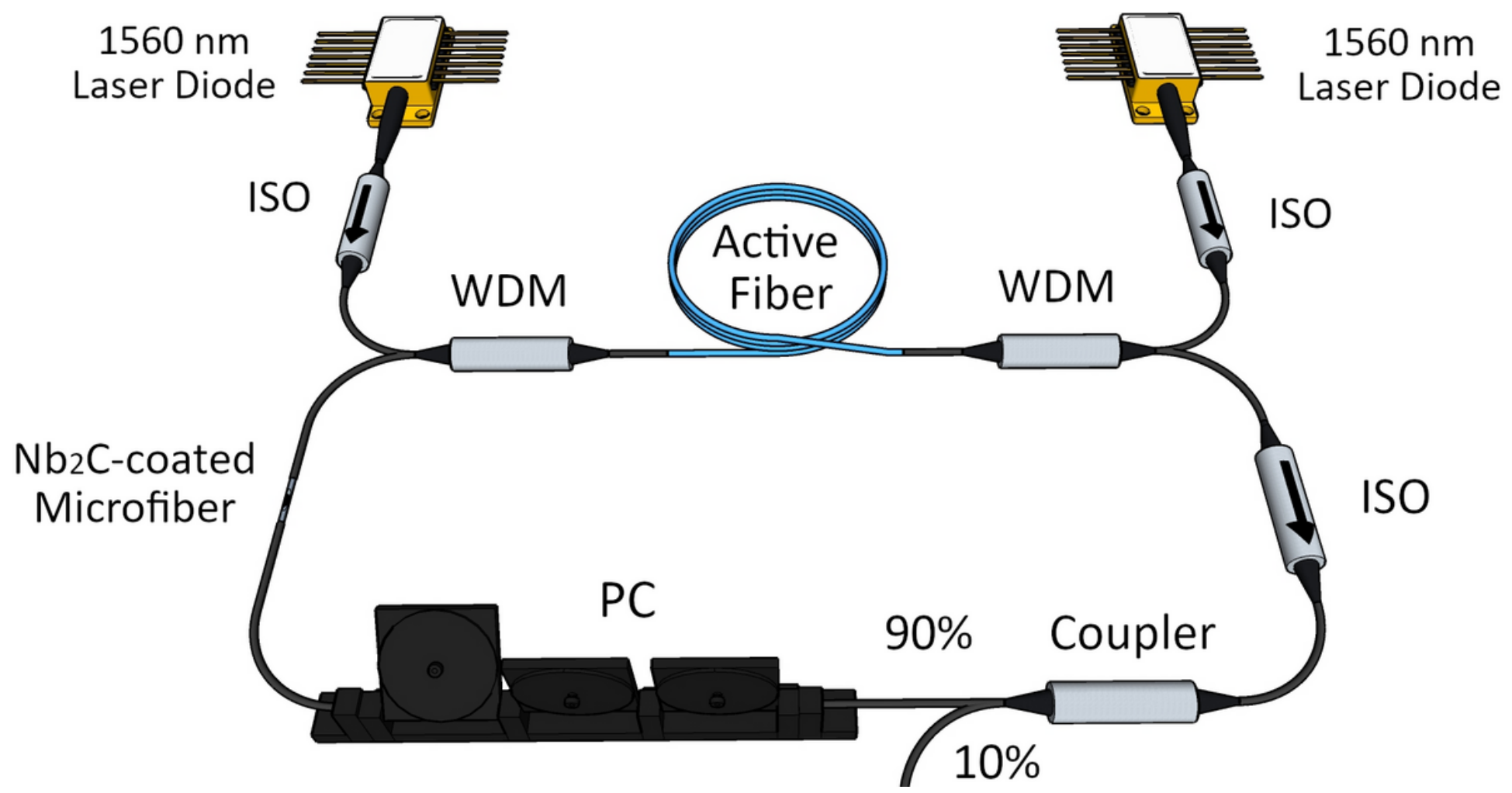

Figure 5

Schematic diagram of $2.0 \mu \mathrm{m}$ passively mode-locked fiber laser cavity. 


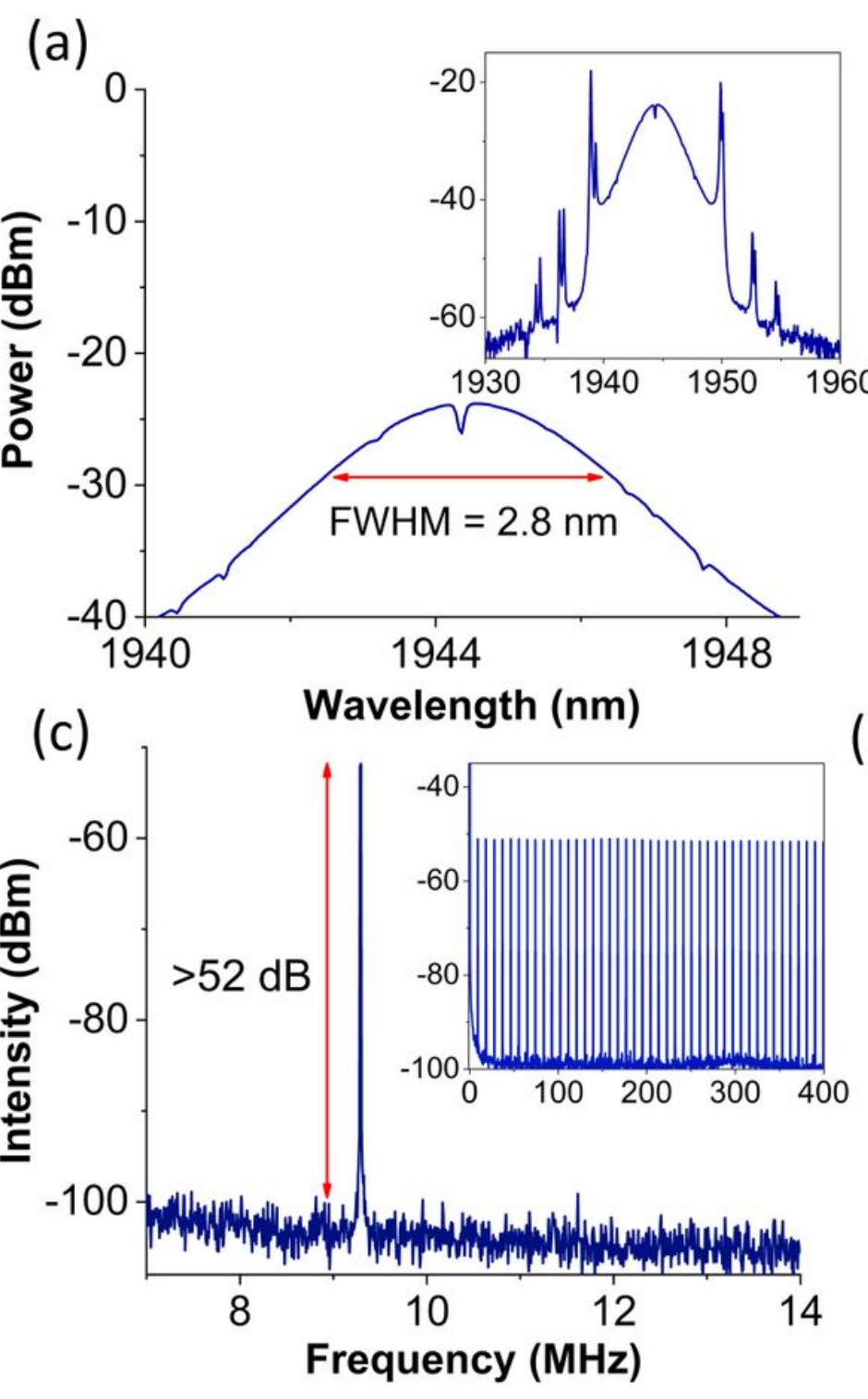

(b)
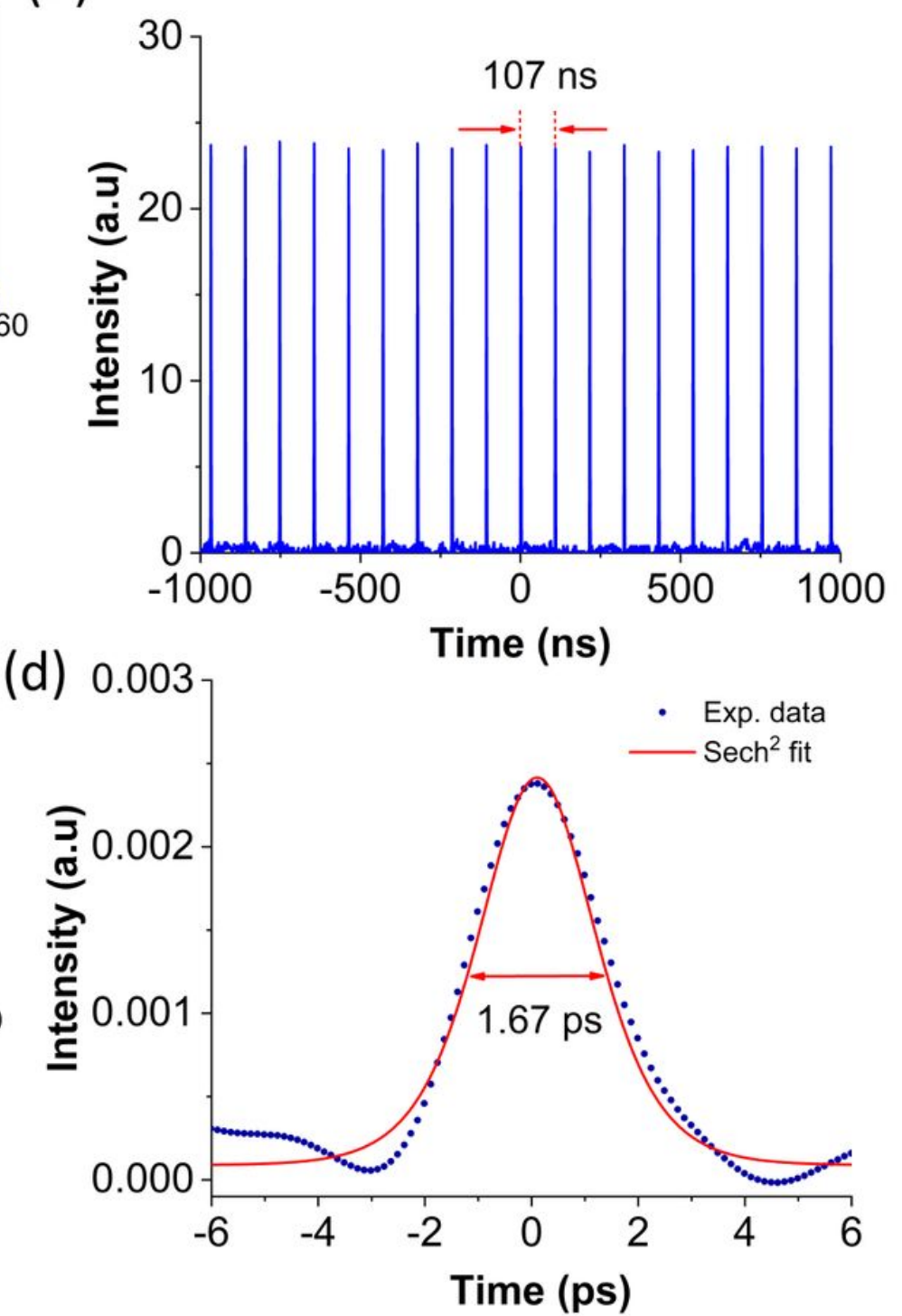

Figure 6

Mode-locked TDFL output at $476 \mathrm{~mW}$. (a) Enlarged optical spectrum (Inset shows full soliton spectrum); (b) oscilloscope trace; (c) autocorrelation trace and; (d) radio frequency (RF) spectrum (Inset shows RF spectrum on wider span range of $400 \mathrm{MHz}$ ). 
(a)
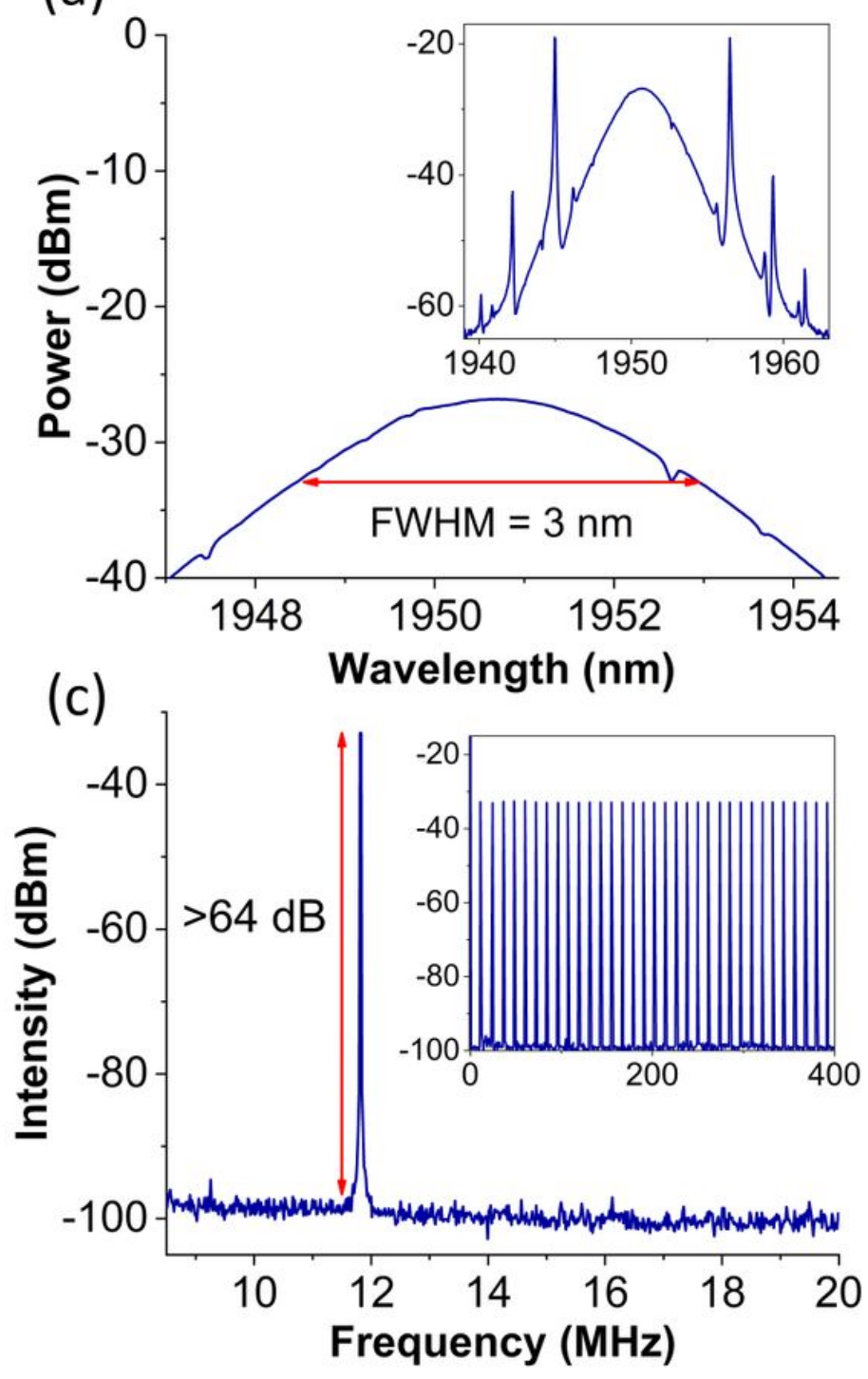

(b)

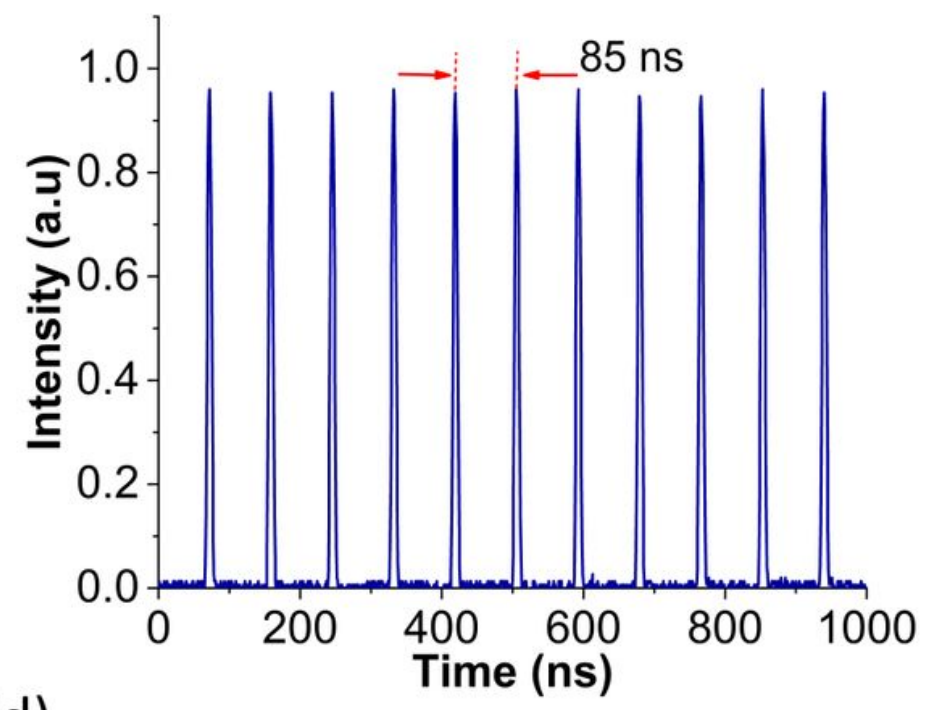

(d)

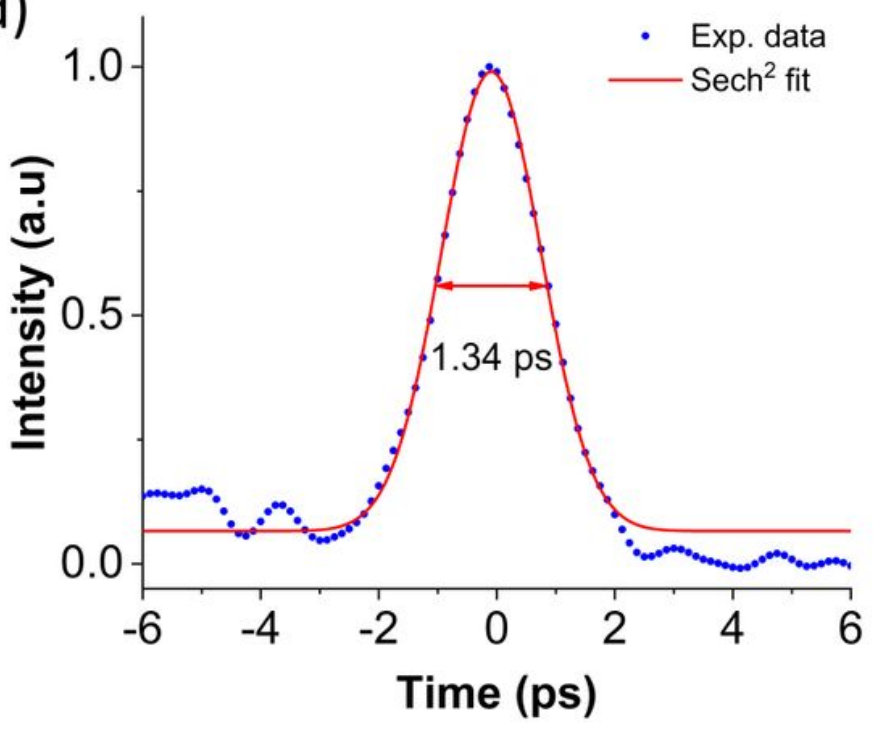

Figure 7

Mode-locked THDFL output at $397 \mathrm{~mW}$. (a) Enlarged optical spectrum (Inset shows full soliton spectrum); (b) pulse train; (c) autocorrelation trace and; (d) radio frequency (RF) spectrum (Inset shows RF spectrum on wider span range of $400 \mathrm{MHz}$ ). 


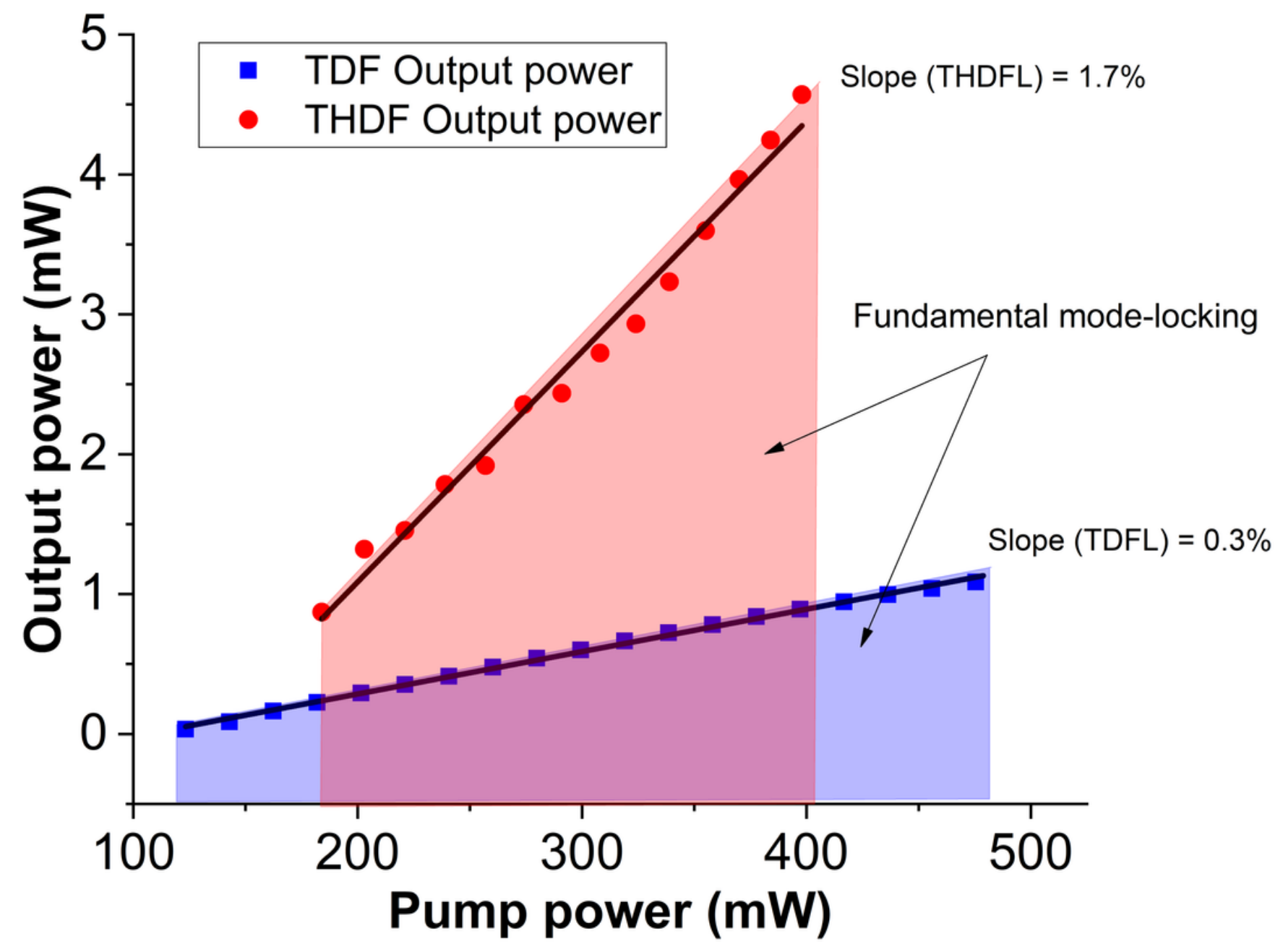

Figure 8

Variation of output power against pump power for TDFL and THDFL.
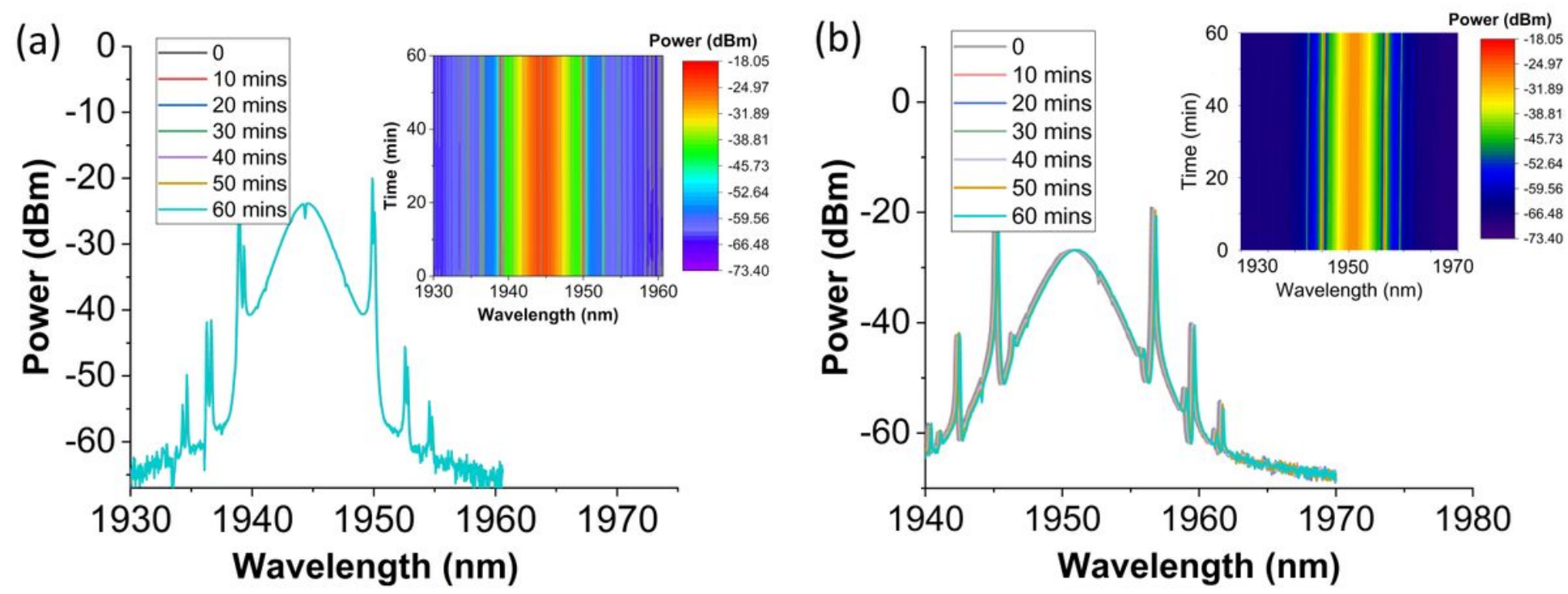
Figure 9

Stability measurement of (a) TDFL at $476 \mathrm{~mW}$ and (b) THDFL at $397 \mathrm{~mW}$ over a period of 1-hour. 